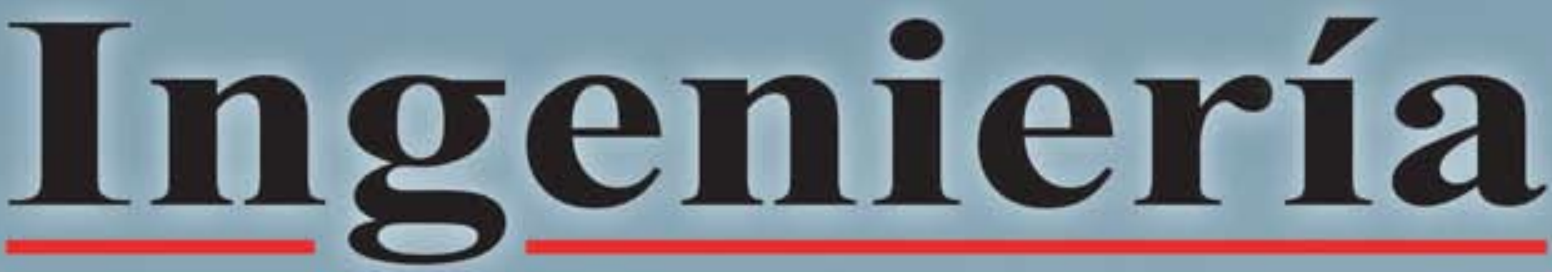

ENERO/DICIEMBRE 2004 - VOLUMEN 14 - N¹ y 2

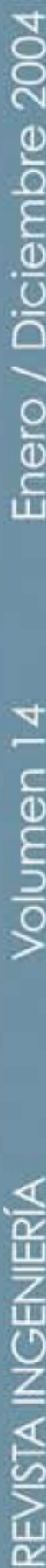
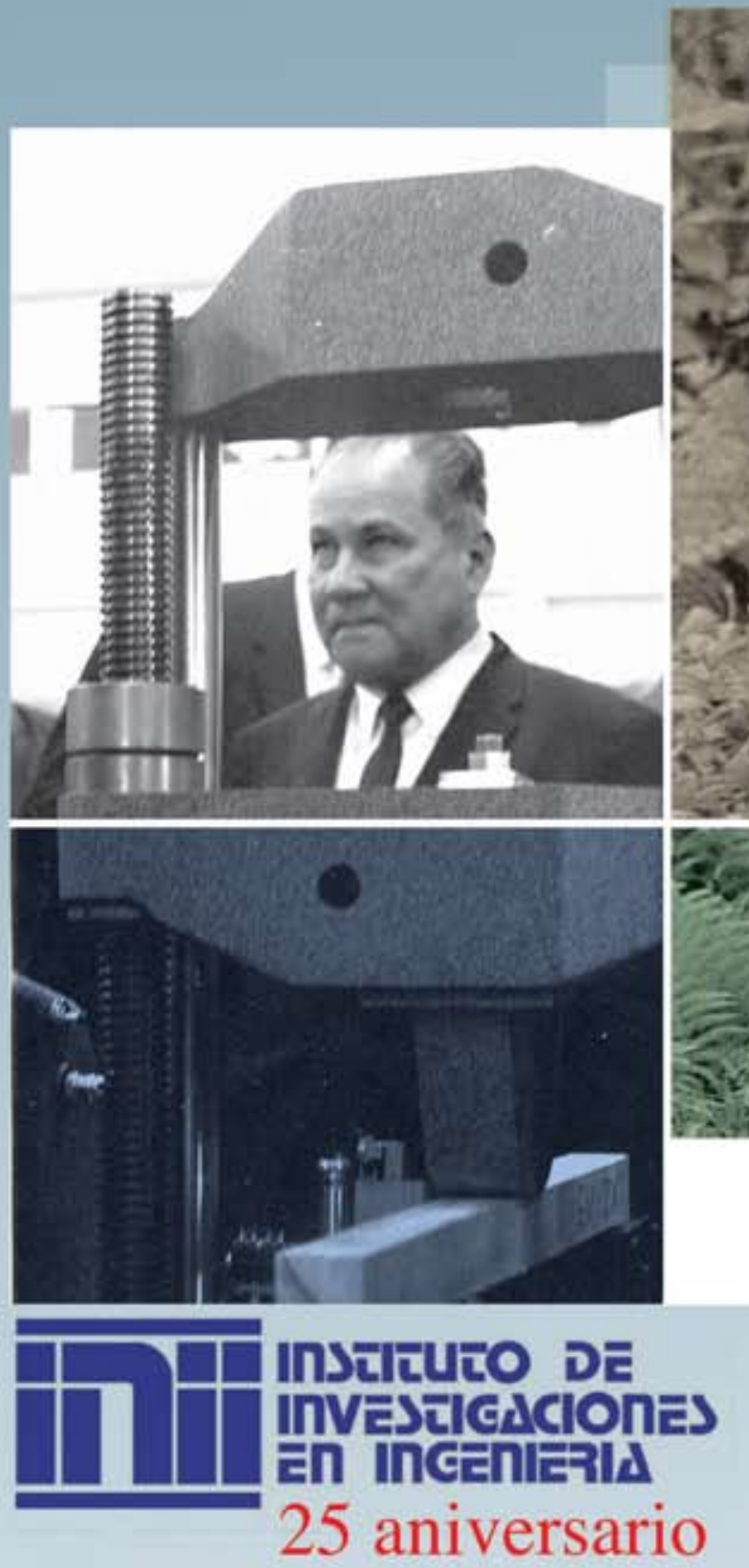

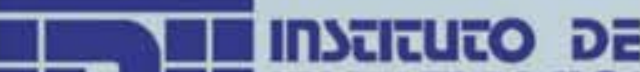
T W Investcscines हn Incenlei|d 25 aniversario

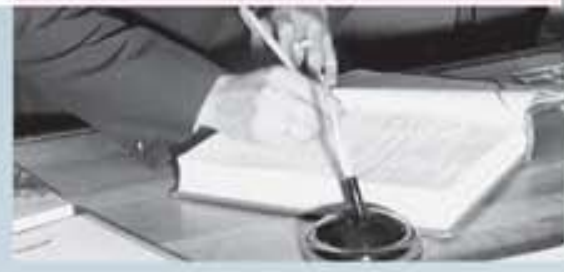




\title{
ESTUDIO COMPARATIVO DE LA PRODUCCIÓN DE ETANOL VÍA FERMENTATIVA UTILIZANDO CUATRO SUSTRATOS PREPARADOS A PARTIR DE BANANO MADURO
}

\author{
Alfredo López Calvo \\ Manuel Molina Córdoba \\ Alberto Huguet Soliva
}

\begin{abstract}
Resumen
Se estudió y se comparó la producción de etanol por fermentación a partir de cuatro sustratos diferentes preparados por hidrólisis de banano maduro. Dichos sustratos fueron la pulpa, la fruta completa, el jugo de la pulpa y el jugo de la fruta. Para la hidrólisis de la pulpa y de la fruta se probaron dos grupos de enzimas: a) $\alpha$-amilasas, $\beta$-amiloglucosidasas y b) pectinasas. Se encontró que la hidrólisis con pectinasas es la que genera mayores rendimientos de jugo, con el menor consumo energético.

En la fermentación se estudió la producción de etanol para ser utilizado como combustible, considerando como variables de diseño el tipo de sustrato, el tiempo de fermentación, la concentración inicial de S.cerevisiae y la tasa de dilución del sustrato. Además, se dio seguimiento cinético a la fermentación en un volumen de $5 \mathrm{~L}$ durante $35 \mathrm{~h}$. Los rendimientos de etanol más altos se obtuvieron mediante la fermentación de los sustratos de pulpa y fruta. El rendimiento máximo fue de $0,0742 \mathrm{~L}$ de etanol $/ \mathrm{kg}$ de banano de la fruta completa.
\end{abstract}

Palabras clave: Etanol, fermentación de banano maduro, pulpa de banano como sustrato, S.cereviceae.

\begin{abstract}
The ethanol production by fermentation of four differents substrates prepared by hydrolysis of ripe bananas was studied. Pulp, whole fruit, pulp juice and fruit juice were the substrates studied. Two groups of enzymes were used in the hydrolysis of the pulp and the fruit: a) $\alpha$-amylases, $\beta$-amiloglucosidases and b) pectinases. It was found that pectinolitic hydrolysis produced higher juice yields with lower energy costs.

Ethanol production through fermentation was studied considering as design variables the type of substrate, the fermentation time, the initial concentration of $S$. cereviceae yeast and the dilution ratio. Fermentation kinetics was also monitored during $35 \mathrm{~h}$ in a $5 \mathrm{~L}$ batch reactor. The higher ethanol yields were obtained by fermenting pulp and fruit substrates. The maximum ethanol yield obtained was $0,0742 \mathrm{~L} / \mathrm{kg}$ of whole fruit.
\end{abstract}

Keywords: Ethanol, ripe banana fermentation, substrate pulp banana, S. cereviceae.

Recibido 04-III-04 • Aceptado 14-X-04

\section{INTRODUCCIÓN}

En Costa Rica, la actividad bananera constituye una de las principales fuentes de divisas de nuestra economía, al ocupar el segundo lugar dentro de los productos de exportación. Sin embargo, las altas exigencias de calidad de los mercados, así como las restricciones en ellos, provocan un excedente no exportable, el cual llega a alcanzar hasta un $12 \%$ de la producción total.
Actualmente de un $50 \%$ a un $60 \%$ de este excedente se utiliza en industrias nacionales para la producción de puré congelado y jugo de banano, entre otros, mientras que del $40 \%$ al $50 \%$ restante se desecha, generando graves problemas de contaminación. Se estiman disponibilidades de banano de rechazo de hasta 80000 toneladas por año (Vong,1996).

El aprovechamiento de desechos agrícolas ha sido objeto de estudio (Boruff, 1961; Duckworth, 
1966; Sitton, 1982; Víquez, 1995; López, 1998); en el caso particular del banano, es importante por representar volúmenes considerables con posibilidades de ser explotados. Dado que la fruta de banano maduro posee un bajo costo, una disponibilidad inmediata sin necesidad de grandes inversiones para su recolección, y además contiene una significativa cantidad de azúcares (cerca del $20 \%$ ), en este trabajo se estudia la posibilidad de producir alcohol mediante la fermentación de la fruta, para su utilización en la formulación de combustibles.

Con este propósito, esta investigación tuvo por objetivo estudiar y comparar la producción de etanol por fermentación a partir de cuatro sustratos (fuentes de carbono) preparados por hidrólisis de banano maduro.

\section{MATERIALES Y MÉTODOS}

\subsection{Materiales}

Como materia prima se utilizó banano Musa cavendishii de grado de maduración 6, según la escala de Chacón et al. (1987), con una razón de pulpa/banano de 0,59 , un 23,8 \% de sólidos totales, un contenido de 7,1 \% de sacarosa, 5,2 $\%$ de glucosa, 3,2 \% de fructosa y $0,64 \%$ de cenizas. La fruta provenía de la zona atlántica y se compró en el CENADA, Barreal de Heredia. Las enzimas empleadas en la hidrólisis de la fruta fueron la Thermamyl ( $\alpha$-amilasa), Amiloglucosidasa ( $\beta$-glucosidasa) y Pectinex Ultra SP-L ( pectinolítica ), de la casa comercial Novo Industries.

Se utilizó el método colorimétrico de Nelson Somogyi (Southgate, 1976) para la determinación de azúcares reductores y totales, el método enzimático de Trinder para la cuantificación de glucosa. El análisis de contenido de azúcares de la materia prima fue realizado por los laboratorios del CITA, mediante HPLC, utilizando una columna de aminex HPX87C.

\subsection{Pretratamiento del banano maduro}

El objetivo del pretratamiento del banano fue desactivar las enzimas causantes del oscurecimiento y preservar de esta manera el sustrato en buenas condiciones para su hidrólisis y fermentación. Este consistió en escaldar la fruta por $15 \mathrm{~min}$ con vapor de agua a $96{ }^{\circ} \mathrm{C}$. A continuación se molió para obtener un puré fino. Si la hidrólisis enzimática no se realizaba seguidamente, entonces el puré se empacaba en cantidades de aproximadamente $1 \mathrm{~kg}$ en bolsas de polietileno de alta densidad y se almacenaba en una cámara $\mathrm{a}-30{ }^{\circ} \mathrm{C}$.

\subsection{Hidrólisis enzimática del banano maduro}

El objetivo de esta primera etapa fue seleccionar el tipo de enzima más adecuado para la preparación de los sustratos a utilizar en la etapa fermentativa. La variable de diseño fue el tipo de enzima, siendo el primero el par constituido por la $\alpha$-amilasa y la amiloglucosidasa y el segundo tipo fue la enzima pectinolítica. Las variables de respuesta fueron el consumo de potencia en la agitación durante la hidrólisis, el contenido de azúcares finales, tanto totales como reductores y el rendimiento de jugo.

Inicialmente, se colocó una masa de aproximadamente $1 \mathrm{~kg}$ de puré en el recipiente destinado para la hidrólisis. Mediante un baño térmico se elevó la temperatura del puré hasta la necesaria, para la aplicación de la enzima. Al adicionar la enzima, se empezó a registrar la variación del torque en el equipo, el cual se relaciona matemáticamente con la potencia consumida y la velocidad de agitación. En el Cuadro 1 se muestran las condiciones en las cuales se trabajaron las diferentes enzimas. 


\subsection{Preparación de los sustratos}

Seleccionado el tipo de enzima para el proceso de hidrólisis, se procedió a la preparación de los diferentes sustratos. Para el caso de los sustratos semisólidos ( pulpa y fruta) se procedió a hidrolizar el puré obtenido del pretratamiento térmico mediante la aplicación de la enzima pectinolítica, utilizando las condiciones indicadas en el Cuadro 1. La diferencia entre estos dos sustratos es la presencia de las cáscaras molidas en el sustrato denominado "fruta".

Al finalizar el tiempo de hidrólisis se empacó aproximadamente $1 \mathrm{~kg}$ del hidrolizado en bolsas de polietileno de alta densidad y se pasteurizó a $90{ }^{\circ} \mathrm{C}$ por $10 \mathrm{~min}$. La preparación y determinación de los rendimientos de los jugos requirió de la extracción de los hidrolizados de la fruta y pulpa, lo cual se realizó con ayuda de una prensa hidráulica operando a una presión de $65 \mathrm{kPa}$. La preservación de estos sustratos se realizó de la misma manera que con los sustratos semisólidos.

\subsection{Estudio de la fermentación}

El objetivo de esta segunda etapa fue determinar aquella combinación de tipo de sustrato, razón de dilución, concentración inicial de levaduras y tiempo de fermentación, que proporcionara el rendimiento más alto de etanol, expresado éste como volumen de etanol producido por masa de banano empleado.
La cepa de S.cerevisiae fue facilitada por la Fábrica Nacional de Licores ( FANAL ) y se cultivó en una incubadora de cama oscilante a una temperatura de $31{ }^{\circ} \mathrm{C}$ y utilizando el $\mathrm{pH}$ natural de la fruta, el cual se encuentra alrededor de 4,5 .

Para llevar a cabo las fermentaciones, se seleccionó como diseño experimental un cuadrado grecolatino de cuatro variables y un número de réplicas de dos. En el Cuadro 2 se muestra el diseño experimental utilizado.

\subsection{Seguimiento cinético de la fermentación}

El objetivo para esta tercer etapa fue determinar la variación con respecto al tiempo de algunas variables de importancia en procesos fermentativos, tales como: concentración de azúcares, biomasa, concentración de etanol, $\mathrm{pH}$ y oxígeno disuelto. Con esta información se determinaron algunos parámetros cinéticos de la fermentación alcohólica del sustrato de banano.

Se utilizaron los siguientes parámetros para la fermentación a una escala de $5 \mathrm{~L}$ :

- Fuente de carbono: se utilizó la pulpa con la cáscara previamente hidrolizada (PC) como fuente de azúcares para la fermentación, debido a que proporciona los mejores rendimientos en la producción de etanol.

- Condiciones ambientales: se trabajó a una temperatura de (30 a 32) ${ }^{\circ} \mathrm{C}$ y al $\mathrm{pH}$ natural de la fruta que se encuentra entre 4,5 y 4,8 .

Cuadro 1. Condiciones recomendadas para la aplicación de las enzimas en la hidrólisis del banano maduro

$\begin{array}{lccc}\text { Enzima } & \alpha \text { amilasa } & \beta \text { glucoamilasa } & \text { Pectinasa } \\ \text { Concentración }(\mathrm{g} / \mathrm{kg} \text { sustrato) } & 1,0 & 1,0 & 0,5 \\ \text { Tiempo de acción (min) } & 60 & 60 & 60 \\ \text { Temperatura }\left({ }^{\circ} \mathrm{C}\right) & 93 & 60 & 38-40 \\ \mathrm{pH} & 6,5 & 4,5 & 4,5\end{array}$

Fuente: (Hammond,1996; Víquez,1995) 
- Concentración inicial de levaduras: se utilizó una concentración de $10^{8}$ de células/ $\mathrm{mL}$ de medio de cultivo.

- Tiempo de fermentación: se fijó en 35 h.

- Medio anaerobio: de experimentos previos (Cysewsky, 1976; Vong, 1996) se encontró una mejor respuesta a la producción de alcohol cuando se evacuó inicialmente el oxígeno del medio mediante burbujeo con nitrógeno, razón por lo que se utilizó esta forma de trabajo.

- Agitación: se utilizó una agitación de $250 \mathrm{rpm}$; una razón diámetro de propela a diámetro de recipiente de 0,35 .

- Dilución: se utilizó una tasa de dilución de $0,4 \mathrm{~kg}$ de agua/kg de sustrato, según recomienda Hammond, (1996).

\section{RESULTADOS Y DISCUSIÓN}

En la Figura 1 se muestra el comportamiento del consumo de potencia en la agitación durante la hidrólisis del puré de banano, para los dos tipos de enzimas estudiados: amilasas y pectinasas. Al comparar las curvas de potencia consumida de ambas enzimas se observa que las pectinasas originan en general un consumo menor que las amilasas. Además, las pectinasas necesitan una temperatura de trabajo $\left(45^{\circ} \mathrm{C}\right)$ inferior que las amilasas $\left(90{ }^{\circ} \mathrm{C}\right.$ y $60{ }^{\circ} \mathrm{C}$ ), lo cual disminuye la cantidad de energía requerida para el calentamiento, en forma considerable; además, su acción es mucho más rápida, lo que provoca una disminución sustancial de la viscosidad lo que se manifiesta en un menor requerimiento de potencia. .

En el Cuadro 3 se muestran los resultados para el contenido de azúcares y rendimiento de jugo que proporcionaron las diferentes enzimas a los hidrolizados de pulpa y fruta. Todos los valores representan promedios de determinaciones obtenidas por triplicado.

El rendimiento de jugo es claramente superior cuando se utiliza la enzima pectinolítica y la concentración de azúcares totales es ligeramente superior para las amilasas.

Debido a que la enzima pectinasa proporciona un menor consumo de potencia durante la hidrólisis, tanto de agitación como de calentamiento, presenta poca diferencia en el contenido de azúcares totales en relación con las amilasas, se obtiene un mayor rendimiento de jugo, y tiene

Cuadro 2. Diseño experimental para la fermentación alcohólica

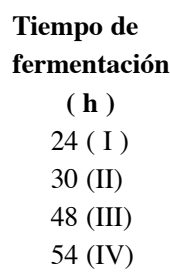

Variables estudiadas en el cuadrado grecolatino

$\begin{array}{cccc}\begin{array}{c}\text { Tiempo de } \\ \text { fermentación } \\ \text { ( h ) }\end{array} & \text { Tipo de sustrato } & \begin{array}{c}\text { Concentración inicial de levaduras } \\ \text { (millones células/mL) }\end{array} & \begin{array}{c}\text { Dilución } \\ \text { (kg agua/kg sustrato) }\end{array} \\ 24 \text { ( I ) } & \text { Fruta, PC (1) } & 25(\mathrm{~A}) & 0,2(\alpha) \\ 48 \text { (II) } & \text { Pulpa, P (2) } & 50(\mathrm{~B}) & 0,4(\beta) \\ 54 \text { (IV) } & \text { Jugo fruta, JPC (3) } & 75(\mathrm{C}) & 0,6(\delta) \\ & \text { Jugo pulpa, JP (4) } & 100(\mathrm{D}) & 0,8(\gamma)\end{array}$

La variable de respuesta de los experimentos se expresa como $\mathrm{L}$ de etanol (medidos a $20^{\circ} \mathrm{C}$ ) por $\mathrm{kg}$ de fruta empleada $(\mathrm{L} / \mathrm{kg})$.

$\begin{array}{lcccc} & \text { Sustrato } 1 & \text { Sustrato } 2 & \text { Sustrato } 3 & \text { Sustrato } 4 \\ \text { Tiempo I } & \text { A } \alpha & \text { B } \gamma & \text { C } \delta & \text { D } \beta \\ \text { Tiempo II } & \text { B } \beta & \text { A } \delta & \text { D } \gamma & \text { C } \alpha \\ \text { Tiempo III } & \text { C } \gamma & \text { D } \alpha & \text { A } \beta & \text { B } \delta \\ \text { Tiempo IV } & \text { D } \delta & \text { C } \beta & \text { B } \alpha & \text { A } \gamma\end{array}$




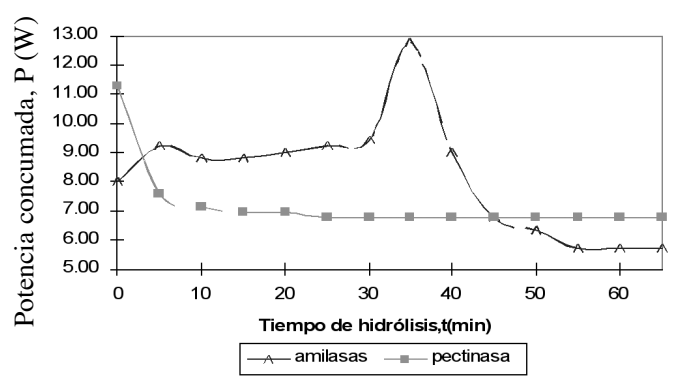

Figura 1. Variación de la potencia consumida por el agitador durante la hidrólisis enzimática de la pulpa.

un costo similar. Se concluye que el proceso de hidrólisis del banano maduro debe realizarse con este tipo de enzimas, para obtener los mejores resultados con el menor costo. Realizada la selección del tipo de enzima se prepararon los diferentes sustratos para la etapa fermentativa; en el Cuadro 4 se muestra la caracterización de estos.

Las fermentaciones se realizaron siguiendo el diseño experimental mostrado en el Cuadro 2. En el Cuadro 5 se presentan los rendimientos de alcohol obtenidos y en el Cuadro 6, los resultados estadísticos. Si se observa la magnitud del valor de $\mathrm{F}$ experimental para las distintas fuentes de variación, y se les compara con el valor de $\mathrm{F}_{(3 / 16)}$ para un $95 \%$ de confianza en una prueba de dos colas: 3,24 (Box, Hunter y Hunter, 1978), se encuentra que tanto el tiempo de fermentación, el tipo de sustrato así como la concentración inicial de levaduras proporcionan resultados significativamente diferentes en sus distintos niveles. De forma contraria, las razones de dilución utilizadas no afectan los resultados en forma significativa. Se encuentra, además, que la interacción entre las variables no es significativa, por lo que se pueden estudiar en forma independiente en futuros experimentos.

Puesto que la variable sustrato es significativa para determinar cuál o cuáles de los distintos niveles estudiados de dicha variable genera resultados diferentes, se debe recurrir a la distribución de referencia móvil (Box, Hunter y Hunter, 1978). En la Figura 2 se muestra la distribución de referencia, se observa cómo los sustratos semisólidos originan resultados que caen en la misma curva de distribución, al igual que los jugos; sin embargo, la curva no agrupa simultáneamente a los dos grupos, lo que los hace diferentes.

La variable sustrato presentó un rendimiento de etanol mayor cuando se fermentaron los sustratos pulpa (P) y fruta (PC), mientras que los jugos, aunque producían mostos más concentrados en etanol, presentaron rendimientos respecto a banano total empleado mucho menores, porque los rendimientos en la extracción del jugo no fueron bastante altos.

Cuadro 3. Concentración de azúcares y rendimiento de jugo de los hidrolizados de banano maduro.

\begin{tabular}{|c|c|c|c|}
\hline & $\begin{array}{c}\text { Azúcares } \\
\text { Reductores } \\
\mathbf{C}_{\mathbf{R}} \\
(\%)\end{array}$ & $\begin{array}{c}\text { Azúcares } \\
\text { Totales } \\
\mathbf{C}_{\mathbf{T}} \\
(\%)\end{array}$ & $\begin{array}{c}\text { Rendimiento } \\
\text { de jugo } \\
\mathbf{R}_{\mathbf{J}} \\
\text { (base fruta) }\end{array}$ \\
\hline \multicolumn{4}{|l|}{ HIDROLIZADO } \\
\hline Fruta/amilasas & 10,6 & 24,3 & 48 \\
\hline Fruta/pectinasas & 7,6 & 19,0 & 59 \\
\hline Pulpa/amilasas & 12,4 & 27,9 & 30 \\
\hline Pulpa/pectinasas & 10,8 & 27,0 & 39 \\
\hline \multicolumn{4}{|c|}{ SIN HIDROLIZAR } \\
\hline Fruta & 6,0 & 15,8 & \\
\hline Pulpa & 8,5 & 22,6 & \\
\hline
\end{tabular}


Cuadro 4. Sustratos de banano maduro empleados para la fermentación alcohólica.

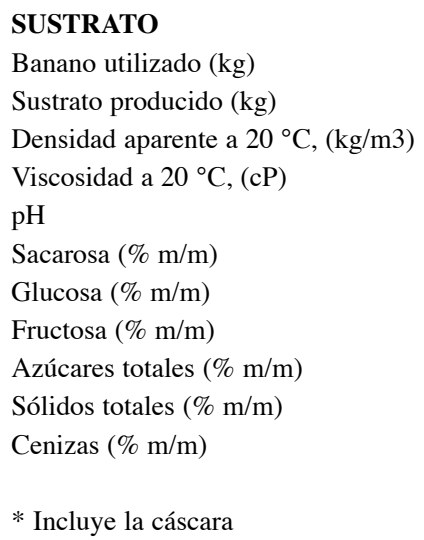

* Incluye la cáscara

$\begin{array}{cc}\text { PULPA } & \text { FRUTA* } \\ 45,0 & 35,0 \\ 29,0 & 25,0 \\ 1086,0 & 1064,0 \\ 850,0 & 5500,0 \\ 4,63 & 4,79 \\ 7,1 & 6,6 \\ 5,2 & 3,4 \\ 3,1 & 2,3 \\ 15,4 & 12,3 \\ 23,8 & 20,5 \\ 0,64 & 0,79\end{array}$

JUGO PULPA

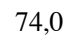

21,0

1078,0

2,9

4,70

10,3

3,9

3,6

17,8

$-$
JUGO FRUTA

66,0

31,0

1054,0

2,0

4,84

7,8

3,0

2,8

13,6
20,5
0,79

Cuadro 5. Resultados de rendimientos utilizando el diseño experimental grecolatino duplicado.

\begin{tabular}{|c|c|c|c|c|}
\hline Tiempo & Sustrato 1 & Sustrato 2 & Sustrato 3 & Sustrato 4 \\
\hline \multirow[t]{3}{*}{ I } & $\mathbf{A} \alpha$ & B $\gamma$ & $\mathrm{C} \delta$ & $\mathbf{D} \beta$ \\
\hline & 0,0518 & 0,0495 & 0,0242 & 0,0412 \\
\hline & 0,0408 & 0,0466 & 0,0356 & ,0368 \\
\hline \multirow[t]{3}{*}{ II } & B $\beta$ & A $\delta$ & $\mathbf{D} \gamma$ & $\mathrm{C} \alpha$ \\
\hline & 0,0225 & 0,0405 & 0,0317 & 0,0264 \\
\hline & 0,0374 & 0,0304 & 0,0396 & 0,0238 \\
\hline \multirow[t]{3}{*}{ III } & $\mathbf{C} \gamma$ & $\mathbf{D} \alpha$ & $\mathbf{A} \beta$ & B $\delta$ \\
\hline & 0,0620 & 0,0447 & 0,0271 & 0,0305 \\
\hline & 0,0579 & 0,0598 & 0,0242 & 0,0282 \\
\hline \multirow[t]{3}{*}{ IV } & D $\delta$ & $\mathbf{C} \beta$ & $\mathbf{B} \alpha$ & $\mathbf{A} \gamma$ \\
\hline & 0,0638 & 0,0477 & 0,0280 & 0,0261 \\
\hline & 0,0617 & 0,0542 & 0,0333 & 0,0325 \\
\hline
\end{tabular}

Cuadro 6. Análisis estadístico del cuadrado grecolatino

$\begin{array}{lcccc}\text { Fuente devariación } & \begin{array}{c}\text { Suma decuadrados } \\ (\text { SS })\end{array} & \begin{array}{c}\text { Grados libertad } \\ (v)\end{array} & \text { Cuadrado promedio } & \text { Valor F } \\ \text { Tiempo } & 685,4 & 3 & 228,5 & 7,0 \\ \text { Sustrato } & 2524,6 & 3 & 841,5 & 25,7 \\ \text { Concentración inicial } & & & & \\ \text { de levaduras } & 958,5 & 3 & 319,5 & 9,8 \\ \text { Dilución } & 195,8 & 3 & 65,3 & 2,0 \\ \text { Interacción } & 17,7 & 3 & 5,9 & 1,8 \\ \text { Error } & 523,1 & 16 & 32,7 & - \\ \text { Total } & 5065 & 31 & - & -\end{array}$




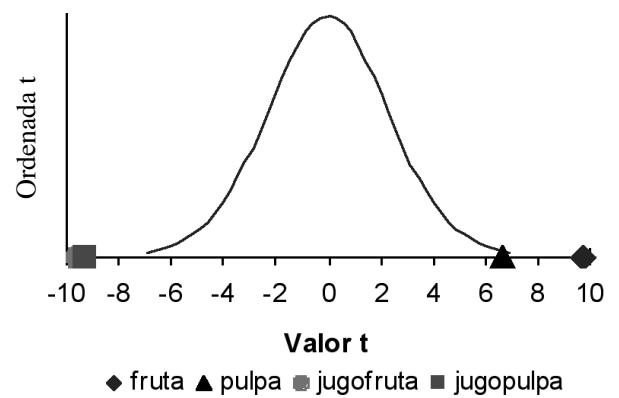

Figura 2. Distribución de referencia móvil para la variable sustrato.

Para el tiempo de fermentación, no se encontró diferencia significativa entre los niveles estudiados, por lo que se recomienda tomar un tiempo de fermentación corto, generalmente de $30 \mathrm{~h}$ a $35 \mathrm{~h}$.

Al incrementarse la concentración inicial de levaduras en el medio se incrementó el rendimiento de etanol, por lo que se seleccionó la concentración de levaduras mayor, es decir, 100 millones de células por mililitro. Respecto a la razón de dilución, como no se encontró tampoco diferencia significativa entre sus niveles, se seleccionó el valor de $0,4 \mathrm{~kg}$ agua $/ \mathrm{kg}$ sustrato, el cual es un valor recomendado para este tipo de sustratos (Hammond, 1996).

Para las fermentaciones a mayor escala (5 L) estas se realizaron por duplicado para dar seguimiento cinético a las diferentes variables de interés. Para tal propósito, se utilizó la fruta completa. En la Figura 3 se muestra el comportamiento de la concentración ponderada de etanol, azúcares reductores y glucosa con respecto al tiempo.

Durante las primeras horas de la fermentación se presenta una producción creciente de etanol, hasta que se alcanza una etapa de poca producción que corresponde al agotamiento de las reservas alimenticias de las levaduras.

La concentración máxima obtenida fue de 4,2 $\% \mathrm{~m} / \mathrm{m}$ de etanol; el metabolismo de la $S$. cerevisiae se favorece en un medio de mayor carácter anaeróbico (Cysewsky,1976; Sitton,

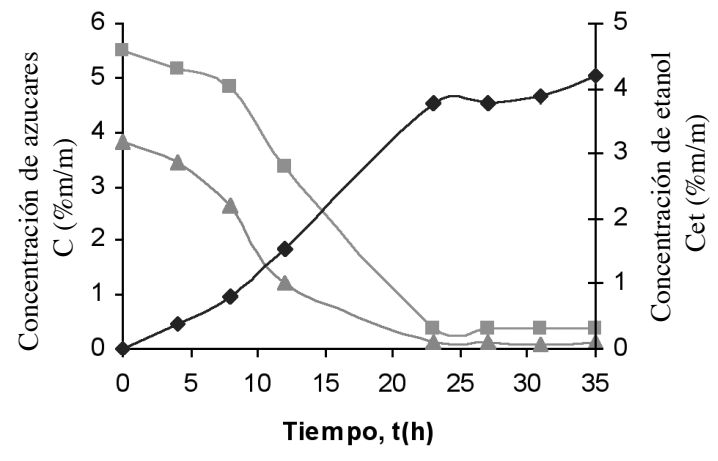

$\neg$ Azucar. Reduct. $\longleftarrow$ Glucosa $\multimap$ Conc.etanol.

Figura 3. Consumo de azúcares y producción de etanol respecto al tiempo a una escala de $5 \mathrm{~L}$.

1982; Amato,1992; Hammond, 1996; Bu'lock, J.y Kristiansen. B., 1987).

Para el consumo de azúcares reductores y glucosa durante la fermentación, se observa cómo el agotamiento de las reservas de azúcares coincide con la etapa de poca producción alcohólica. El efecto del agotamiento de los azúcares disponibles para la fermentación también se aprecia en el comportamiento de la concentración de biomasa. Debido a la ausencia de suficiente alimento, las levaduras detienen su proceso reproductivo de carácter exponencial, tal como se muestra en la Figura 4. Es claro que a partir de las $20 \mathrm{~h}$ a las $25 \mathrm{hs}$ de fermentación, cuando queda una concentración residual de azúcares, las levaduras dejan de reproducirse.

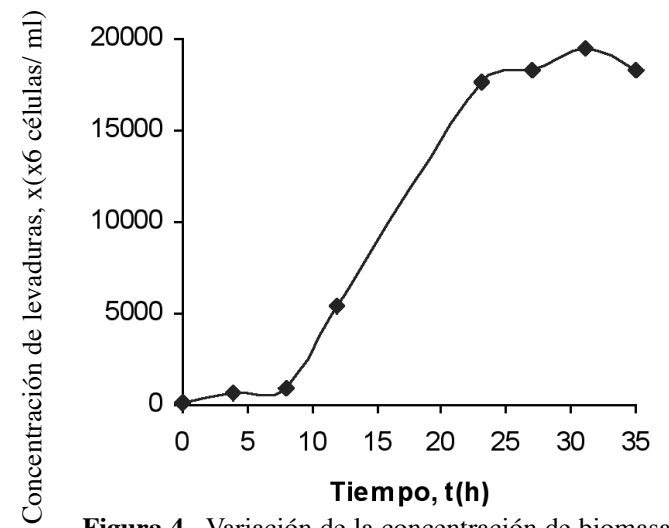

Figura 4. Variación de la concentración de biomasa durante la fermentación a escala de $5 \mathrm{~L}$. 
En la Figura 5 se muestra el comportamiento de $\mathrm{pH}$ con respecto al tiempo para la fermentación en estudio. La disminución del pH se debió a la producción de metabolitos ácidos provenientes de otras rutas metabólicas de la levadura, o bien, por la contaminación del mosto con bacterias acéticas u otro microorganismo.

En la Figura 6 se muestra el comportamiento del oxígeno disuelto respecto al tiempo, éste decrece conforme avanza la fermentación, debido a que una fracción del total de levaduras lo consume como parte de la vía metabólica aeróbica, característica de los anaerobios facultativos como la S. cerevisiae.

En el Cuadro 7 se muestran los parámetros que caracterizan la cinética del proceso fermentativo para este tipo de sustratos (corrida II), obtenidos a partir de los resultados del seguimiento cinético de las fermentaciones a escala de 5L, los cuales son comparados con parámetros obtenidos cuando el medio de cultivo no fue previamente burbujeado con nitrógeno (corrida I), encontrándose una diferencia sustantiva en los rendimientos y productividades, lo que hace evidente la importancia de eliminar oxígeno previamente.

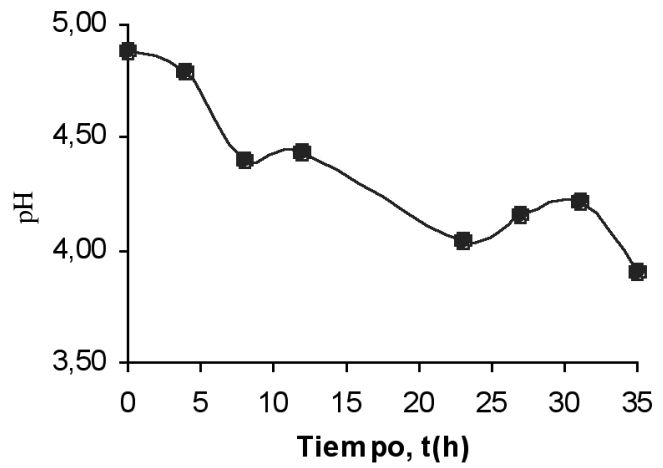

Figura 5. Variación del $\mathrm{pH}$ respecto al tiempo para la fermentación a una escala de $5 \mathrm{~L}$.

\section{Resumen del proceso}

En la Figura 7 se muestra un resumen de la información obtenida en las diferentes etapas experimentales desarrolladas en este trabajo y que, representa el proceso más adecuado para la producción de etanol a partir de la fruta completa de banano maduro.

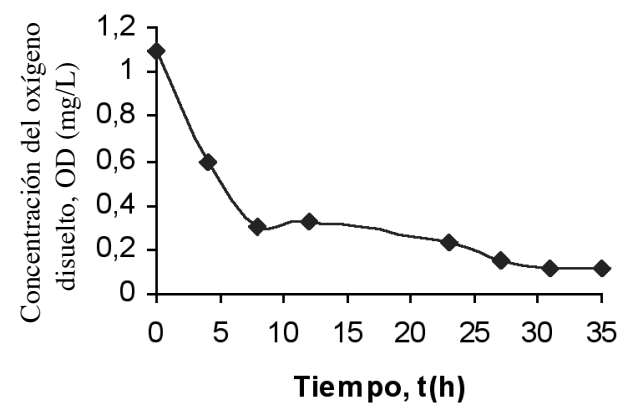

Figura 6. Disminución del oxígeno disuelto para la fermentación a escala de $5 \mathrm{~L}$.

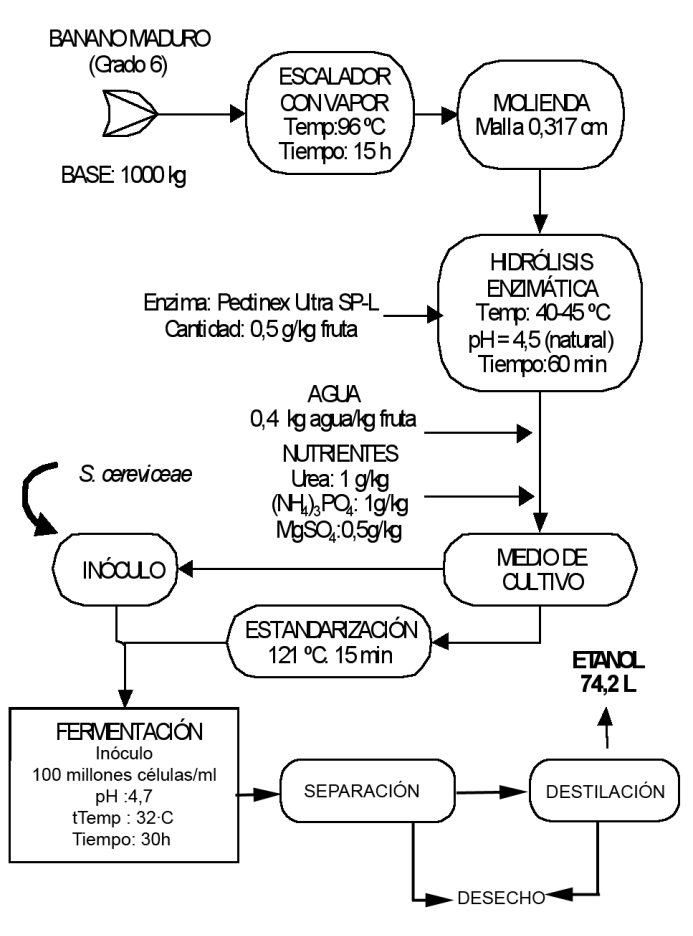

Figura 7. Diagrama para la producción de etanol a partir de banano maduro por vía fermentativa. 
Cuadro 7. Parámetros cinéticos para las fermentación alcohólica a escala de 5L utilizando el sustrato preparado por hidrólisis de la fruta completa.

\section{PARÁMETROS CINÉTICOS}

Tiempo máximo de fermentación, $\mathrm{t}(\mathrm{h})$

Tiempo para obtener máxima concentración de etanol, $\mathrm{t}(\mathrm{h})$

Rendimiento técnico del etanol, (L/kg fruta)

Concentración final de etanol, P (g de etanol/kg sustrato)

Productividad del etanol, P (g de etanol/kg de sustrato/h)

Azúcares consumidos, S (g azúcares $/ \mathrm{kg}$ de sustrato)

Rendimiento del etanol, Yp/s (g/g azúcar consumido)

Rendimiento del etanol con respecto al teórico

Tasa específica de producción de etanol, Qp ( $\mathrm{g}$ etanol/célula/h)

Rendimiento de biomasa, Yx/s ( cél./g azúcares consumido)

Tasa específica de producción de biomasa, Qx ( cél./g azúcares consumidos/h )

Tasa específica de consumo de azúcar, Qs (g azúcar/células/h)

$\frac{d C_{e \tan o l}}{d t}$, tasa de producción de etanol en la fase logarítmica, $(\%$ $\mathrm{m}$ etanol/h)

$\frac{d S}{d t}$, tasa de consumo de azúcares reductores en la fase logarítmica, $(\% \mathrm{~m} / \mathrm{h})$

\section{CORRIDA I CORRIDA II}

35 35

23

0,0484

0,0742

27,28

1,19

84,61

84,87

0,32

0,49

0,63

0,96

$8,5 E-14$

$1,0 E-13$

$1,6 E+11$

$2,1 E+11$

$7,1 E+09$

$9,0 E+09$

$2,6 E-13$

$2,09 E-13$

$0,1306 \quad 4,6 E-5 \cdot X$

$r^{2}=0,97 \quad r^{2}=0,99$

$$
\begin{array}{cl}
1,3 E-4 \cdot X & 6,5 E-5 \cdot X \\
r^{2}=0,99 & r^{2}=0,98
\end{array}
$$

\section{CONCLUSIONES}

1. El escaldado con vapor a $96^{\circ} \mathrm{C}$ durante $15 \mathrm{~min}$ proporciona una desactivación satisfactoria de las enzimas causantes del pardeamiento enzimático del banano maduro. Aunque la desactivación no es total, es aceptable para el proceso propuesto.

2. El consumo energético por calentamiento y agitación es menor cuando se utilizan enzimas pectinolíticas en el proceso de hidrólisis.
3. Además, la hidrólisis pectinolítica proporciona mayores rendimientos de jugo, expresado éste como volumen de jugo por masa de banano empleado en su producción.

4. El rendimiento de etanol obtenido por fermentación, expresado como L de etanol por $\mathrm{kg}$ de banano utilizado, es significativamente mayor para los sustratos semisólidos preparados a partir de la hidrólisis de la pulpa y de la fruta completa. 
Por lo que no es recomendable la extracción del jugo y posterior fermentación.

5. Bajo las condiciones experimentales estudiadas, el rendimiento de etanol aumenta con la concentración inicial de levaduras en el medio y no se ve afectado por la tasa de dilución.

6. Del estudio de la cinética de la fermentación a mayor escala, se concluye que la concentración de etanol alcanza su máximo cerca de las $24 \mathrm{~h}$, lo cual coincide con el agotamiento de la reserva de azúcares.

7. El rendimiento de etanol más alto que se obtuvo fue de $0,074 \mathrm{~L} / \mathrm{kg}$ de banano para $35 \mathrm{~h}$ de fermentación, el cual se aproxima al valor reportado por Hammond (1996) que es de $0,09 \mathrm{~L} / \mathrm{kg}$ de banano con $72 \mathrm{~h}$ de fermentación.

\section{AGRADECIMIENTOS}

- Al Centro Nacional de Ciencia y Tecnología de Alimentos (CITA), por las facilidades para la preparación de los sustratos de banano y en los análisis químicos.

- Al Dr. Franklin Jiménez y a la señora Dagmar Utzinger, del Laboratorio de Bacteriología Médica de la Facultad de Microbiología, por el préstamo del equipo y el asesoramiento brindado en el manejo del microorganismo.

- A la Fábrica Nacional de Licores (FANAL), por cedernos una muestra de la cepa de $S$. cerevisae. A la compañía Trisán SA, por facilitar la enzima pectinolítica.

\section{BIBLIOGRAFÍA}

AMATO, S. (1992). Procesos fermentativos. Universidad Nacional. Costa Rica.

BORUFF, C. S. (1961). Alcohol industrial. In Kirk, R. E. \& Othmer, R. F. Eds. Enciclopedia de Tecnología Química. UTHEA, México. v.1. p.749-770.

BOX, G., W. \& HUNTER, J. (1978). Statics for experiments. John Wiley \& Sons, New York.

BU'LOCK, J. \& KRISTIANSEN. B. (1987). Basic biotechnology. Academic Press, London.

CHACÓN, S. et al. (1987). Escala fisico-química de maduración del banano, Fruit, 42 (2) 95.

CYSEWSKY, G. R. (1976). Fermentation kinetics and process economics for the production of ethanol. University of California, California.

DUCKWORTH, R. B. (1966). Fruit and vegetables. Pergamon Press, Oxford.

HAMMOND, J.B. (1996). Alcohol from bananas. Bioresource Technology, 56: 125-130.

LÓPEZ, A. (1998). Estudio comparativo de la producción de etanol vía fermentiva utilizando cuatro sustratos preparados a partir de banano maduro, Tesis de Licenciatura en Ingeniería Química. Universidad de Costa Rica, Escuela de Ingeniería Química, San José.

SITTON, O. C. (1982). Ethanol production from agricultural residues. In Mc Ketta, J. J. y Cunningham, W. A., eds. Encyclopedia of Chemical Processing and Design. Mercer Dekker, New York. V.20, p.41-60.

SOUTHGATE, D. A. (1976). Determination of food carbohydrates. Applied Science Publisher, London.

VÍQUEZ, F. R. (1995). Obtención de jugo clarificado de banano en el nivel de planta piloto. Reviteca. 4:10-

VONG, A. (1996). Estudio de la producción de etanol mediante la fermentación de tres sustratos diferentes obtenidos a partir del banano. Tesis de Lic. En Ingeniería Química. Universidad de Costa Rica, Escuela de Ingeniería Química, San José. 


\section{SOBRE LOS AUTORES}

\section{Sc. Alberto Huguet Soliva}

Profesor pensionado de Escuela de Ingeniería Química de la Universidad de Costa Rica.

Teléfono: 272-0448

Correo electrónico: ahuguet@ fing.ucr.ac.cr

\section{Lic. Alfredo López Calvo}

Universidad Houston Texas, USA.

\section{Ing. Manuel Molina Córdoba}

Profesor asociado, Escuela de Ingeniería Química Teléfono: 207-4620 Facsimil: 225-5622

Correo electrónico: emolina@terraba.ing.ucr.ac.cr 